\title{
Licor funcional a base de umbu (Spondias tuberosa Arruda) e microalga (Spirulina
}

\author{
spp.) \\ Functional liquor based on umbu (Spondias tuberosa Arruda) and microalgae (Spirulina spp.) \\ Licor funcional a base de umbu (Spondias tuberosa Arruda) y microalgas (Spirulina spp.)
}

Recebido: 04/03/2021 | Revisado: 11/03/2021 | Aceito: 14/03/2021 | Publicado: 21/03/2021

\author{
João Paixão dos Santos Neto \\ ORCID: https://orcid.org/0000-0003-4645-6866 \\ Instituto Nacional de Investigação Agrária e Veterinária, Portugal \\ E-mail: joaopaixaoneto@gmail.com \\ Yada Maria Pereira dos Santos \\ ORCID: https://orcid.org/0000-0002-5453-1005 \\ Instituto Federal de Educação, Ciência e Tecnologia de Alagoas, Brasil \\ E-mail: yadasantos15@gmail.com \\ Pamela Stephany Jennings Cunha \\ ORCID: https://orcid.org/0000-0002-4534-5278 \\ Universidade Estadual de Mato Grosso do Sul, Brasil \\ E-mail: pamelajennings18@hotmail.com \\ Victória Carolline do Moraes Gatti \\ ORCID: https://orcid.org/0000-0002-7400-1685 \\ Universidade Federal Rural da Amazônia, Brasil \\ E-mail: victoriagatti.agro@gmail.com \\ Henrique da Silva Barata \\ ORCID: https://orcid.org/0000-0001-6356-4629 \\ Universidade Federal Rural da Amazônia, Brasil \\ E-mail: henriquebarata2000@gmail.com \\ Gabriel Gustavo Tavares Nunes \\ ORCID: https://orcid.org/0000-0003-0572-1731 \\ Universidade Federal Rural da Amazônia, Brasil \\ E-mail: eng.agro.gmonteiro@gmail.com \\ Job Teixeira de Oliveira \\ ORCID: https://orcid.org/0000-0001-9046-0382 \\ Universidade Federal do Mato Grosso do Sul, Brasil \\ E-mail: job.oliveira@hotmail.com \\ Fábio Israel Martins Carvalho \\ ORCID: https://orcid.org/0000-0002-8995-2141 \\ Universidade Federal Rural da Amazônia, Brasil \\ E-mail: fabioimc@yahoo.com.br \\ Priscilla Andrade Silva \\ ORCID: https://orcid.org/0000-0002-2774-3192 \\ Universidade Federal Rural da Amazônia, Brasil \\ E-mail: priscilla.andrade@ufra.edu.br \\ Maria Cecília Castelo Branco de Santana \\ ORCID: https://orcid.org/0000-0002-1482-0347 \\ Instituto Federal de Educação, Ciência e Tecnologia de Alagoas, Brasil \\ E-mail: ceciliacastelob@gmail.com
}

\section{Resumo}

O umbu é uma fonte vegetal muito importante para a Caatinga, por possuir compostos importante para alimentação. A microalga Spirulina spp. tem uso como suplemento alimentar funcional. No presente estudo, objetivou-se elaborar e avaliar as características físico-químicas dos licores das folhas e dos frutos de umbu, acrescidos de microalga Spirulina spp., obtidos no Instituto Federal de Alagoas, localizado no município de Piranhas - AL. Foram elaboradas quatro formulações de licor da folha do umbu e quatro formulações de licor da polpa do umbu, adicionados de microalga Spirulina spp. Em seguida, foi realizada a caracterização física de 20 amostras de frutos através das medidas de massa dos frutos, comprimento, diâmetro, densidade e rendimento da polpa. Para a caracterização físico-química dos licores, foram realizadas as análises de $\mathrm{pH}$, acidez total titulável, sólidos solúveis, umidade e cinzas. Os licores das folhas de umbu e polpa de umbu apresentaram os teores médios de pH entre 2,93 a 3,01, respectivamente. Entre as formulações, podemos perceber que a umidade foi elevada, ficando acima de 90\%. A partir destes resultados, observou-se a capacidade destes licores no processamento agroindustrial, pois possuem elevados rendimentos. E que a inclusão de microalga (Spirulina spp.) potencializa o valor nutricional do licor.

Palavras-chave: Caatinga; Suplemento alimentar; Elaboração; Caracterização. 


\begin{abstract}
The umbu is an especially important plant source for the Caatinga, as it has important compounds for food. Spirulina $s p p$. is used as a functional food supplement. In the present study, the objective was to elaborate and evaluate the physical-chemical characteristics of the liqueurs of the leaves and fruits of umbu plus Spirulina spp. obtained at the Federal Institute of Alagoas, located in the municipality of Piranhas - AL. Four formulations of liquor from the umbu leaf and four formulations of liquor from the umbu pulp were added, added by Spirulina spp. Then, the physical characterization of 20 fruit samples was carried out by measuring the fruit's mass, length, diameter, density, and pulp yield. For the physical-chemical characterization of the liquors, analyzes of $\mathrm{pH}$, total titratable acidity, soluble solids, moisture, and ashes were carried out. The liqueurs of umbu leaves and umbu pulp showed average $\mathrm{pH}$ levels between 2.93 to 3.01, respectively. Among the formulations, we can see that the humidity was high, getting more than $90 \%$. From these results, the capacity of these liquors in agro-industrial processing was observed, as they have high yields. And that the inclusion of Spirulina spp. enhances the nutritional value of the liquor, with high sensory acceptance by potential consumers.
\end{abstract}

Keywords: Caatinga; Food supplement; Elaboration; Description.

\title{
Resumen
}

Umbu es una fuente vegetal muy importante para la Caatinga, ya que tiene compuestos importantes para la alimentación. Spirulina spp. se utiliza como complemento alimenticio funcional. En el presente estudio, el objetivo fue elaborar y evaluar las características físico-químicas de los licores de las hojas y frutos de umbu más Spirulina spp. obtenido en el Instituto Federal de Alagoas, ubicado en el municipio de Piranhas - AL. Se agregaron cuatro formulaciones de licor de la hoja de umbu y cuatro formulaciones de licor de la pulpa de umbu, agregadas por Spirulina spp. Luego, se realizó la caracterización física de 20 muestras de frutos midiendo la masa, longitud, diámetro, densidad y rendimiento de pulpa de los frutos. Para la caracterización físico-química de los licores se realizaron análisis de $\mathrm{pH}$, acidez total titulable, sólidos solubles, humedad y cenizas. Los licores de hojas de umbu y pulpa de umbu mostraron niveles de $\mathrm{pH}$ promedio entre 2,93 y 3,01, respectivamente. Entre las formulaciones, podemos ver que la humedad era alta, llegando a más del 90\%. A partir de estos resultados, se observó la capacidad de estos licores en el procesamiento agroindustrial, ya que tienen altos rendimientos. Y que la inclusión de Spirulina spp. realza el valor nutricional del licor, con una alta aceptación sensorial por parte de los consumidores potenciales.

Palabras clave: Caatinga; Suplemento alimenticio; Elaboración; Descripción.

\section{Introdução}

O umbuzeiro, frutífera endêmica da Caatinga, ocorre naturalmente apenas no Semiárido brasileiro. A espécie Spondias tuberosa Arruda é conhecida como "imbu”, "ambu” ou "umbu”, que em tupi-guarani significa "árvore que dá de beber". O nome é uma alusão aos frutos suculentos e às suas raízes, ou túberas, que parecem grandes batatas, onde se acumula água e nutrientes para sustentar a planta durante o período de estiagem, responsável pela complementação de renda de muitas famílias de agricultores do Semiárido (Drumond et al., 2016).

$\mathrm{O}$ umbu apresenta atividade antioxidante que desempenham o sequestro de espécies reativas de oxigênio (radicais livres), pela presença de compostos fenólicos $\left(90,4 \pm 2,2 \mathrm{mg} .100 \mathrm{~g}^{-1}\right)$ e vitamina $\mathrm{C}\left(19,5 \pm 1,8 \mathrm{mg} \cdot 100 \mathrm{~g}^{-1}\right.$ de polpa), na literatura existem dados também relatando a presença de flavonoides, antocianinas e carotenoides (Almeida et al., 2011; Ramalho et al., 2011; Rufino et al., 2010). Segundo Gomes et al. (2011), detectaram a presença de compostos fenólicos de baixo peso molecular. Em análises por Cromatógrafo Líquido Ultra Rápido encontraram a presença de ácido gálico, clorogênico $\left(8,5 \mu \mathrm{g} \cdot \mathrm{g}^{-1} \mathrm{de}\right.$ polpa $)$ foi o majoritário, protocatecóico, pcumárico, vanílico e ferrulíco, os quais possuem propriedades terapêuticas. No estudo do extrato das folhas do umbuzeiro (S. tuberosa) apresentou a presença de ácido elágico, flavonóis, quercetina e rutina os quais desempenham atividade antiviral contra o vírus da dengue tipo 2 (Silva, 2011).

A microalga Spirulina spp. é uma cianobactéria que realiza fotossíntese convertendo os nutrientes do meio em material celular, liberando oxigênio (Colla, 2002), pode ser adicionada em alimentos preparados na quantidade de 0,5 a 3,0 g porção-1 (FDA, 2003). Em maio de 2009, a microalga Spirulina spp., passou a fazer parte da Lista de Novos Ingredientes (enquadrada nos Alimentos com Alegações de Propriedades Funcionais e/ou de Saúde, Novos Alimentos/Ingredientes, Substâncias Bioativas e Probióticos) aprovada pela Agência Nacional de Vigilância Sanitária, que limita a sua ingestão diária em 1,6 g por indivíduo (Brasil, 2009). 
Sua utilização na alimentação deve-se à sua composição química, que por sua vez, proporciona efeitos nutricionais e potencialmente funcionais ao consumidor, apresenta em sua constituição 53 - 62\% de proteínas; 17 - 25\% de carboidratos; 4 6\% de lipídios; 8 - 13\% de minerais e 3 - 6\% de umidade (FDA, 2003), na composição nutricional possui a pró vitamina A e a vitamina C, importantes antioxidantes naturais, e ácido fólico (B9), importante para a formação de células e bom funcionamento de alguns órgãos. Esta microalga destaca-se, sobretudo, pelo seu conteúdo de vitamina B12, difícil de encontrar em dietas vegetarianas (Becker, 2004).

A produção artesanal de licores constitui alternativa interessante para proporcionar aumento da renda familiar, pois o processamento exige tecnologia simples, o produto final é comercializado em temperatura ambiente e apresenta extensa vida de prateleira (Barros et al., 2008). Diante do exposto, entende-se que o estudo de métodos para elaboração de licores com alta concentração de compostos bioativos torna-se relevante.

O objetivo deste estudo foi elaborar e caracterizar a composição físico-química e sensorial do licor funcional típico da Caatinga a base de folhas e frutos do umbuzeiro acrescidos de diferentes proporções de microalga Spirulina spp.

\section{Material e Métodos}

As folhas e os frutos do umbuzeiro foram coletados no município de Piranhas, localizado na mesorregião do Sertão Alagoano, tem uma altitude aproximada de $88 \mathrm{~m}$ e coordenadas geográficas de 9॰37'38' de latitude sul e $37^{\circ} 45^{\prime} 25^{\prime \prime}$ ' de longitude oeste.

As folhas e os frutos foram selecionados em estádio maduros provenientes da safra 2018/19. A alga Spirulina spp. em pó foi adquirida em loja de manipulação, localizada em Maceió-Alagoas.

Foi realizada a caracterização física das folhas e dos frutos de umbu, logo após a colheita foram selecionadas e avaliadas 20 amostras de cada fruto e 200 gramas de folhas. Os pesos foram determinados através de balança analítica. A altura e o diâmetro dos frutos foram mensurados por paquímetro. A densidade foi averiguada pelo deslocamento da coluna de água com auxílio de uma proveta.

Foram elaboradas quatro formulações de licor da folha do umbu e quatro formulações de licor da polpa do umbu, adicionados de microalga Spirulina spp., de acordo com a Tabelas 1. Em cada tratamento, realizou-se a infusão através da maceração das folhas e a imersão dos frutos do umbu em cachaça de graduação alcoólica de 37,5\% (v/v) por 20 dias. Depois desse período, as soluções foram filtradas em filtro de nylon e os extratos alcoólicos obtidos foram misturados com xarope de sacarose a $43{ }^{\circ}$ Brix. Adicionaram-se as concentrações de microalga Spirulina spp. em vasilhame tipo inox, previamente sanitizado. Após 60 dias de envelhecimento, realizaram-se a filtragem final dos licores, com o uso de uma bomba à vácuo em frasco tipo kitassato de 1 litro, acoplado por um funil tipo Büchner com filtro de celulose. Em seguida realizou-se a embalagem em garrafas de vidro e armazenadas em temperatura ambiente. 
Tabela 1. Desenvolvimento dos licores com folhas e polpa de umbu com microalga Spirulina spp.

\begin{tabular}{cccc}
\hline \multicolumn{5}{c}{ Formulações dos licores com as folhas de umbu } \\
\hline Tratamento & Folhas do umbu (g) & Cachaça (mL) & Spirulina spp. $(\mathrm{g})$ \\
& & & 0,0 \\
T1 & 400 & 970 & 0,3 \\
T2 & 400 & 970 & 0,9 \\
T3 & 400 & 970 & 1,5 \\
T4 & 400 & 970 & \\
& & & Spirulina spp. $(\mathrm{g})$ \\
\hline Tratamento & Formulações dos licores com a polpa de umbu & 0,0 \\
T1 & Polpa de umbu (g) & Cachaça (mL) & 0,3 \\
T2 & 800 & 970 & 0,9 \\
T4 & 800 & 970 & 1,5 \\
\hline
\end{tabular}

T1 - Controle, T2 - 0,3\% de Spirulina spp., T3 - 0,9\% de Spirulina spp., T4 - 1,5\% de Spirulina spp.; g (gramas), mL (mililitros). Fonte: Autores.

As folhas, a polpa dos frutos e os licores obtidos foram analisados quimicamente quanto ao $\mathrm{pH}$, sólidos solúveis (SS), acidez titulável (AT), todas em triplicatas; umidade e cinzas, em quadruplicata, de acordo com a metodologia descrita pelo Instituto Adolfo Lutz (IAL, 2008).

A análise estatística das variáveis respostas foi realizada com o auxílio do software Agroestat (Barbosa; Maldonado Junior, 2015). Após análise de variância as médias foram comparadas pelo Teste de Tukey, com $p \leq 0,05$.

\section{Resultados e discussão}

\subsection{Caracterização física e química dos frutos e das folhas de umbu}

A caracterização física dos frutos de umbu pode ser visualizada através da Tabela 2. A partir das variáveis físicas dos frutos, ou seja, a massa, o diâmetro, a densidade e o rendimento da polpa, se observa que os valores apresentaram alta variabilidade, principalmente na massa dos frutos. Esta variação pode ser explicada pelo fato de as plantas não possuírem os tratos culturais para o máximo aproveitamento da cultura (Tabela 2).

Os frutos apresentaram tamanhos (diâmetro e comprimento) e massas elevados, uma vez que, a maioria dos frutos in natura que apresentam maior tamanho e massa, são mais apreciados para o consumo, pois os consumidores destinam a preferência à aparência dos frutos mais volumosos (Chitarra \& Chitarra, 2005).

Tabela 2. Caracterização física dos frutos de umbu.

\begin{tabular}{lccccc}
\hline & $\begin{array}{c}\text { Massa } \\
(\mathrm{g})\end{array}$ & $\begin{array}{c}\text { Comprimento } \\
(\mathrm{cm})\end{array}$ & $\begin{array}{c}\text { Largura } \\
(\mathrm{cm})\end{array}$ & $\begin{array}{c}\text { Densidade } \\
\left(\mathrm{g} \mathrm{mL}^{-1}\right)\end{array}$ & $\begin{array}{c}\text { Firmeza } \\
(\mathrm{N})\end{array}$ \\
\hline Média & 20,74 & 3,55 & 2,91 & 1,04 & 70,11 \\
\hline$D P$ & 3,26 & 0,26 & 0,13 & 0,16 & 26,31 \\
\hline
\end{tabular}

g (gramas); cm (centímetros); g/mL (gramas/mililitros); N (Nilton). Fonte: Autores.

Costa et al. (2015), avaliando os aspectos biométricos dos frutos de umbu da Região do Semiárido Brasileiro, relataram valores para comprimento e largura de 2,53 a 4,35 cm e de 2,46 a 4,30 cm, respectivamente. Valores médios para comprimento 
e largura em frutos de umbu, coletados na Região Semiárida do Município de Janaúba - Minas Gerais de 3,41 e 3,47 cm, respectivamente foram verificados por Ferreira et al. (2015).

A caracterização físico-química dos frutos e folhas de umbu colhidos em Piranhas - Alagoas na safra de 2018/2019, estão dispostas nas Tabelas 3 e 4 .

Tabela 3. Composição físico-química das folhas de umbu

\begin{tabular}{lcccccc}
\hline & $\begin{array}{c}\text { Sólidos Solúveis } \\
(\mathrm{SS})\left({ }^{\circ} \text { Brix }\right)\end{array}$ & $\mathrm{pH}$ & $\begin{array}{c}\text { Acidez Titulável } \\
(\mathrm{AT})\left(\mathrm{g} 100 \mathrm{~g}^{-1}\right)\end{array}$ & $\begin{array}{c}\text { Ratio } \\
\left(\mathrm{SS} \mathrm{AT}^{-1}\right)\end{array}$ & $\begin{array}{c}\text { Umidade } \\
\left(\mathrm{g} \mathrm{100g}^{-1}\right)\end{array}$ & $\begin{array}{c}\text { Cinzas } \\
\left(\mathrm{g} \mathrm{100g}^{-1}\right)\end{array}$ \\
\hline Média & 0,10 & 3,07 & 0,20 & 0,50 & 81,35 & 0,11 \\
\hline$D P$ & $4,00 \times 10^{-3}$ & 0,06 & 0,02 & 0,08 & 1,10 & 0,10 \\
\hline
\end{tabular}

DP - Desvio Padrão. Resultados expressos em base úmida. Fonte: Autores.

As características físico-químicas das folhas de umbu, Tabela 3, demonstraram baixos teores de sólidos solúveis (SS) e acidez titulável (AT). Esses resultados implicam diretamente na composição do sabor, pois estão associados à concentração de e açúcares e ácidos presentes nos frutos, principalmente frutose e ácido cítrico. A melhor combinação, mais saborosa, ocorre com altos níveis de ácidos e açúcares. O que não foi observado nos frutos do umbuzeiro do referido trabalho. No entanto, segundo Nascimento-Silva et al. (2008), ao estudarem a histoquímica das folhas de Spondias tuberosa Arruda, evidenciaram a presença de compostos fenólicos, em especial taninos, e alcaloides, estes compostos secundários são de grande importância medicinal e nutracêutica.

O potencial hidrogeniônico $(\mathrm{pH})$ das folhas, Tabela 3, demonstraram que são muito ácidas (3,07), contribuindo para redução da capacidade de desenvolvimento microbiano. Para o teor de umidade, as folhas apresentaram um valor médio elevado. Outro ponto relevante cientificamente é que no extrato das folhas do umbuzeiro, de acordo Silva (2011), foi detectado atividade antiviral contra o vírus da dengue tipo 2, devido à presença de ácido elágico, flavonóis, quercetina e rutina. As folhas possuem baixo teor de sólidos solúveis, assim como uma baixa relação de $\mathrm{SS} \mathrm{AT}^{-1}$ (ratio). A umidade apresentou alto valor médio e as cinzas baixo teor médio nas folhas do umbuzeiro.

Tabela 4. Composição físico-química dos frutos de umbu.

\begin{tabular}{|c|c|c|c|c|c|c|}
\hline & $\begin{array}{l}\text { Sólidos Solúveis } \\
\text { (SS) ( }\left({ }^{\circ} \text { Brix) }\right.\end{array}$ & $\mathrm{pH}$ & 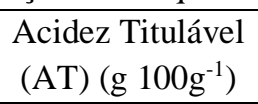 & $\begin{array}{c}\text { Ratio } \\
\left(\mathrm{SS} \mathrm{AT}^{-1}\right)\end{array}$ & 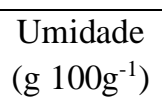 & $\begin{array}{c}\text { Cinzas } \\
\left({\left.\mathrm{g} 100 \mathrm{~g}^{-1}\right)}\right.\end{array}$ \\
\hline Média & 11,13 & 2,79 & 0,78 & 14,33 & 90,19 & 0,27 \\
\hline$\overline{D P}$ & 0,01 & 0,02 & 0,008 & 0,10 & 0,55 & 0,03 \\
\hline
\end{tabular}

DP - Desvio Padrão. Resultados expressos em base úmida. $(\mathrm{n}=3)$. Fonte: Autores.

A Tabela 4 demonstra as características dos frutos de umbu. Para o parâmetro sólidos solúveis (SS) nota-se uma média de $11,13^{\circ}$ Brix, esse valor associa-se ao estádio de maturação maduro. Na literatura outros valores médios de sólidos solúveis, como por exemplo, $8,50^{\circ} \mathrm{Brix}$ (Bastos et al., 2016), 9,70 ${ }^{\circ}$ Brix (Oliveira et al., 2008) e 10,70 ${ }^{\circ}$ Brix (Moura et al., 2013). Conforme Costa et al. (2015), frutos com maior massa apresentam maior teor de sólidos solúveis, ou seja, são mais doces.

O potencial hidrogeniônico $(\mathrm{pH})$ é uma característica importante do fruto, uma vez que pode influenciar o tempo de conservação, ao evitar o desenvolvimento de microrganismos e influenciar a atividade enzimática, não alterando o sabor-odor (“flavor") de produtos de frutas, entre outros efeitos (Lima et al., 2013). O pH da polpa dos frutos de umbu demonstra que é um 
alimento muito ácido (2,79), Passos et al. (2013), encontrou em sua pesquisa, valores em torno de 4,43 e 3,62, sendo assim, os valores estão na faixa que contribui para a redução da capacidade de desenvolvimento microbiano.

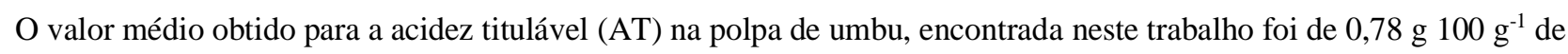
ácido cítrico, parâmetro este importante na apreciação do estado de conservação dos frutos. Ferreira et al. (2015), observaram valores de AT entre 0,41 a 0,58 g $100 \mathrm{~g}^{-1}$ em frutos de Spondias tuberosa, já Pinheiro et al. (2015), averiguaram valores entre 1,00 e 1,50 g $100 \mathrm{~g}^{-1}$ nos diferentes estádios de maturação para frutos da mesma espécie, resultados inferiores aos obtidos neste estudo. A partir destes resultados, os autores inferiram que os parâmetros avaliados se diferenciaram com as variações edáficas.

O ratio (relação SS $\mathrm{AT}^{-1}$ ) indica o balanço entre ácidos e açúcares; logo, quando os valores desta relação são altos, significa que o fruto está com elevado grau de maturação, pois esse grau aumenta quando há decréscimo de acidez e elevação no teor de sólidos solúveis, processo este decorrente da maturação (Chitarra \& Chitarra, 2005). Observou-se a média de 14,33 para o ratio da polpa de umbu, em questão. Segundo Moura et al. (2017), o valor médio do ratio de 55 genótipos de umbu do Banco de Germoplasma da Embrapa Semiárido foi de 17,30.

Como é possível observar na Tabela 4, os frutos de umbu podem ser considerados como alimento do tipo úmido, pois

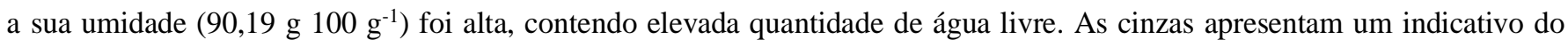
resíduo mineral.

\subsection{Caracterização físico-química dos licores produzidos}

Os parâmetros de qualidade físico-química das diferentes formulações de licor elaborados com as folhas de umbu e Spirulina spp, estão dispostos na Tabela 5. Os valores médios obtidos para os parâmetros avaliados, umidade, extrato seco, cinzas, acidez titulável (ATT), sólidos solúveis (SS) e pH diferiram (p < 0,05) entre as quatro formulações de licores (Tabela 5).

Tabela 5. Teores médios da composição físico-química de licor das folhas de umbu com microalga Spirulina spp.

\begin{tabular}{|c|c|c|c|c|c|c|}
\hline Tratamentos & $\begin{array}{l}\text { Umidade } \\
\left(\mathrm{g} 100 \mathrm{~g}^{-1}\right)\end{array}$ & $\begin{array}{l}\text { Extrato Seco } \\
\qquad\left(\mathrm{g} 100 \mathrm{~g}^{-1}\right)\end{array}$ & $\begin{array}{l}\text { Cinzas } \\
\left(\mathrm{g} 100 \mathrm{~g}^{-1}\right)\end{array}$ & 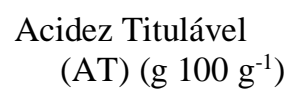 & $\begin{array}{l}\text { Sólidos Solúveis } \\
\text { (SS) ( }{ }^{\circ} \text { Brix) }\end{array}$ & $\mathrm{pH}$ \\
\hline T1 & $91,34 \mathrm{~b}$ & $8,66 \mathrm{a}$ & $0,57 \mathrm{ab}$ & $0,34 \mathrm{a}$ & $16,93 \mathrm{a}$ & $2,93 \mathrm{~b}$ \\
\hline $\mathrm{T} 2$ & $91,83 \mathrm{~b}$ & $8,17 \mathrm{a}$ & $0,43 \mathrm{~b}$ & $0,29 b$ & $17,20 \mathrm{a}$ & $2,99 \mathrm{a}$ \\
\hline $\mathrm{T} 3$ & $91,05 \mathrm{~b}$ & $6,08 \mathrm{~b}$ & $0,54 \mathrm{ab}$ & $0,30 \mathrm{~b}$ & 16,67 a & $3,01 \mathrm{a}$ \\
\hline $\mathrm{T} 4$ & $93,92 \mathrm{a}$ & $8,95 \mathrm{a}$ & $0,80 \mathrm{a}$ & $0,31 \mathrm{~b}$ & $17,67 \mathrm{a}$ & $3,01 \mathrm{a}$ \\
\hline
\end{tabular}

T1 - Controle, T2 - 0,3\% de Spirulina spp., T3 - 0,9\% de Spirulina spp., T4 - 1,5\% de Spirulina spp. Médias seguidas pela mesma letra na mesma linha não diferem estatisticamente entre si pelo teste de Tukey ao nível de 5\% de probabilidade. Resultados expressos em base úmida. $(\mathrm{n}=3)$. Fonte: Autores.

O teor alcoólico médio de $25 \%\left(\mathrm{v} \mathrm{v}^{-1}\right)$ dos licores de folhas e polpas de umbu enquadram-se dentro da faixa estabelecida pela legislação brasileira que é de $15 \%$ a $54 \%$ ( $\left.\mathrm{v} \mathrm{v}^{-1}\right)$ (BRASIL, 2005). Pode-se observar que houve aumento no tratamento T4 em relação ao teor de umidade $(93,92)$, teor de cinzas $(0,80)$ e de sólidos solúveis $(17,67)$, isso possivelmente deve-se a maior concentração de microalga Spirulina spp.

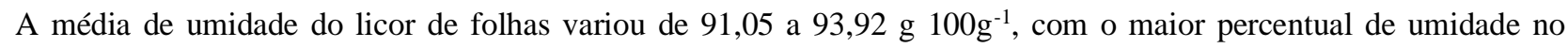
tratamento T4 e diferindo dos demais tratamentos, já o teor médio de cinzas do licor de folhas apresentou maior percentual no T4 e o menor percentual no T2, e, nos T1 e T3 não diferiram entre si. De acordo com Silva et al. (2017), as diferenças quanto a variação de umidade e cinzas podem divergir a partir do trato após a colheita. Os teores de umidade e cinzas na composição físico-química das cascas de tangerina, foram respectivamente $65,40 \%$ e 1,63\%. 
A acidez titulável revelou que o T1 apresenta maior valor médio diferindo estatisticamente dos demais tratamentos. O

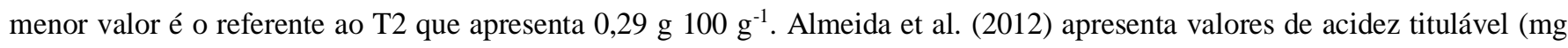
ácido cítrico $100 \mathrm{~g}^{-1}$ ), com o menor valor sendo $0,03 \mathrm{mg}$ ácido cítrico $100 \mathrm{~g}^{-1}$. Os teores de acidez nas cascas e polpas divergem entre si.

Em relação ao conteúdo de sólidos solúveis não houve diferença significativa, sendo que ocorreu uma variação de 16,67 a 17,67 ${ }^{\circ}$ Brix. Esses valores são mais baixos que o valor de sólidos solúveis encontrado no licor de camu-camu por Viera et al. (2010) que apresentou $33^{\circ}$ Brix. Os valores estão dentro do padrão que a legislação determina, valores superiores a $30 \mathrm{~g} \mathrm{~L}^{-1}$.

$\mathrm{O}$ pH médio do licor de folhas de umbu variou de 2,93 a 3,01, o T2, T3 e T4 apresentaram os maiores valores, diferindo significativamente do T1. O pH encontrado para o licor das folhas de umbu (3,01), foi consideravelmente mais baixo que o encontrado por Pereira et al. (2012), obtendo pH de 3,58 para o licor de corte de umbu. O pH baixo está diretamente ligado como fator restritivo para a manifestação de microrganismos patogênicos.

As análises das características físico-química dos licores elaborados com a polpa de umbu estão dispostas na Tabela 6.

Tabela 6. Teores médios da composição físico-química dos licores da polpa de umbu com microalga Spirulina spp.

\begin{tabular}{ccccccc}
\hline Tratamentos & $\begin{array}{c}\text { Umidade } \\
\left(\mathrm{g} \mathrm{100g}^{-1}\right)\end{array}$ & $\begin{array}{c}\text { Extrato Seco } \\
\left(\mathrm{g} \mathrm{L}^{-1}\right)\end{array}$ & $\begin{array}{c}\text { Cinzas } \\
\left(\mathrm{g} 100 \mathrm{~g}^{-1}\right)\end{array}$ & $\begin{array}{c}\text { Acidez Titulável } \\
(\mathrm{AT})\left(\mathrm{g} 100 \mathrm{~g}^{-1}\right)\end{array}$ & $\begin{array}{c}\text { Sólidos Solúveis } \\
(\mathrm{SS})\left({ }^{\circ} \text { Brix }\right)\end{array}$ & $\mathrm{pH}$ \\
\hline T1 & $93,25 \mathrm{a}$ & $6,75 \mathrm{a}$ & $1,33 \mathrm{ab}$ & $0,63 \mathrm{a}$ & $15,13 \mathrm{a}$ & $2,86 \mathrm{a}$ \\
$\mathrm{T} 2$ & $92,90 \mathrm{a}$ & $7,10 \mathrm{a}$ & $1,31 \mathrm{ab}$ & $0,65 \mathrm{a}$ & $14,93 \mathrm{a}$ & $2,86 \mathrm{a}$ \\
$\mathrm{T} 3$ & $92,74 \mathrm{a}$ & $7,20 \mathrm{a}$ & $1,03 \mathrm{~b}$ & $0,66 \mathrm{a}$ & $15,40 \mathrm{a}$ & $2,86 \mathrm{a}$ \\
T4 & $93,03 \mathrm{a}$ & $6,97 \mathrm{a}$ & $1,56 \mathrm{a}$ & $0,66 \mathrm{a}$ & $15,53 \mathrm{a}$ & $2,86 \mathrm{a}$
\end{tabular}

T1 - Controle, T2 - 0,3\% de Spirulina spp., T3 - 0,9\% de Spirulina spp., T4 - 1,5\% de Spirulina spp. Médias seguidas pela mesma letra na mesma linha não diferem estatisticamente entre si pelo teste de Tukey ao nível de $5 \%$ de probabilidade. Resultados expressos em base úmida. $(\mathrm{n}=3)$. Fonte: Autores.

A partir do teste de Tukey a 5\%, observou que somente para as cinzas houve diferença significativa nos tratamentos. A umidade variou de 92,74 a 93,25 g $100 \mathrm{~g}^{-1}$. Os teores de cinzas foram superiores a 1\% para ambos os tratamentos (T1, T2, T3 e T4). De acordo com valores obtidos para o extrato seco disposto na Tabela 6, nem um dos tratamentos possui o teor mínimo para bebida alcoólica mista, a qual preconiza $15 \mathrm{~g} \mathrm{~L}^{-1}$ (Brasil, 2005). As cinzas em alimentos são consideradas como parâmetro de qualidade, pois seus elevados teores retratam maiores teores de cálcio, magnésio, ferro, fósforo, sódio e outros componentes minerais (Wang; Zheng, 2003). As cinzas apresentaram diferença estatística, e o tratamento T4 obteve maior percentual deste constituinte, visto que neste possuía a maior quantidade de microalga Spirulina spp., embora o tratamento T3 apresentasse o menor teor de resíduo mineral, e os tratamentos T1 e T2 não diferiram entre si.

Através da Tabela 6 observa-se os resultados para os teores de sólidos solúveis (SS) dos licores da polpa dos frutos de umbu expresso em ${ }^{\circ} \mathrm{Brix}$. Neste trabalho os tratamentos variam entre 14,93 a $15,53{ }^{\circ} \mathrm{Brix}$. Resultados que apresenta valores mais baixos que os encontrados por Passos et al. (2013) com os valores de 30,0 e 31,0 ${ }^{\circ}$ Brix.

$\mathrm{O} \mathrm{pH}$ dos licores da polpa de umbu apresentaram valor médio de aproximadamente 2,86 para todos os tratamentos, isso representa que todos possuem o caráter ácido.

\section{Conclusão}


Os valores médios das características físico-químicas das folhas e dos frutos de umbu demonstram parâmetros desejáveis para o seu processamento industrial, armazenamento e conservação, devido a sua quantidade significativa de sólidos solúveis, pH e acidez titulável.

Os licores das folhas e dos frutos de umbu, com adição de diferentes porcentagens de microalga Spirulina spp., estão de acordo com a legislação brasileira vigente como mostram as análises físico-químicas.

Realizar a caracterização física e química dos frutos e produtos obtidos, é fundamental para seus usos na indústria de alimentos como na alimentação humana.

Sendo assim, a execução do estudo proposto promoveu avanços no conhecimento sobre o processo agroindustrial de licores artesanais com produtos típicos do Sertão Nordestino. Recomenda-se estudos futuros, na mesma linha, com outros frutos típicos da Caatinga.

\section{Agradecimentos}

Ao Instituto Federal de Alagoas (IFAL, Campus Piranhas). À Universidade Federal Rural da Amazônia (UFRA, Campus de Parauapebas, PA) e à Universidade Federal do Mato Grosso do Sul (UFMS, Chapadão do Sul, MS).

\section{Referências}

Almeida, A. L. S., Albuquerque, U. P. \& Castro, C. C. (2011). Reproductive biology of Spondias tuberosa Arruda (Anacardiaceae), na endemic fructiferous species of the caatinga (dry forest), under different management conditions in northeastern Brazil. Journal of Arid Environments, 75 , 330 - 337. https://.org/10.1016/j.jaridenv.2010.11.003.

Almeida, E. L., Lima, L. C., Borges, V. T. N., Martins, R. N. \& Batalini, C. (2012). Elaboração de licor de casca de tangerina (Citrus reticulata Blanco), variedade ponkan, com diferentes concentrações de casca e tempos de processamento. Alimentos e Nutrição, $23(2), 263$.

Barbosa, J. C. \& Maldonado, J. W. (2015). AgroEstat - sistema para análises estatísticas de ensaios agronômicos. Jaboticabal: FCAV/UNESP. 396 p.

Barros, J. C., Santos, P. A., Isepon, J. S., Silva, J. W. \& Silva, M. A. P. (2008). Obtenção e avaliação de licor de leite a partir de diferentes fontes alcoólicas. Global Science and Technology, 1, 27 - 33.

Bastos, J. S., Martinez, E. A. \& Souza, S. M. A. (2016). Características físico-químicas da polpa de umbu (Spondias tuberosa Arruda Câmara) comercial: efeito da concentração, Journal of Bioenergy and Food Science, 3(1), 11 - 16.

Becker, W. (2004). Microalgae in human and animal nutrition. In: Richmond, A. (Ed). Handbook of microalgal culture: biotechnology and applied phycology. London: Blackwell Science, 312 - 351. 10.1002/9780470995280.

BRASIL (2005). Ministério da Agricultura, Pecuária e Abastecimento. Instrução Normativa n. 13, de 29 de junho de 2005. Aprova o Regulamento Técnico para Fixação dos Padrões de Identidade Qualidade para Aguardente de Cana e para Cachaça. Diário Oficial da União, Brasília, DF, Seção I, página 3

Chitarra, M. I. F. \& Chitarra, A. B. (2005). Pós-colheita de frutos e hortaliças: fisiologia e manuseio. (2a ed.), UFLA.

Colla, L. M., Bertolim, T. E. \& Costa, J. A. V. (2002). Fatty acids profile of Spirulina spp. platensis under different temperatures and nitrogen concentrations. Journal of Biosciences, 59(1), 55 - 59. https://.org/10.1515/znc-2004-1-212.

Costa, F. R., Rêgo, E. R., Rêgo, M. M., Neder, D. G., Silva, S. M. \& Schunemann, A. P. P. (2015). Análise biométrica de frutos de umbuzeiro do Semiárido Brasileiro. Bioscience Journal, Uberlândia, 31(3), 682 - 690. https://.org/10.14393/BJ-v31n3a2015-22844.

Drumond, M. A., Aidar, S. T., Nascimento, C. E. \& Oliveira, V. R. (2016). Umbuzeiro: avanços e perspectivas. Petrolina: Embrapa Semiárido.

FDA - Food and Drug Administration (2003). http://www.fda.gov/Food/FoodIngredientsPackaging/GenerallyRecogn izedasSafeGRAS/GRASListings/ucm153674.htm. ID FDA-2003-N-0161-0006.

Ferreira, L. B., Duarte, A. B., Martins, J. C., Ferreira, A. F. \& Mizobutsi, G. P. (2015). Caracterização Físico-Química de Frutos de Umbuzeiro (Spondias tuberosa Arr. Câm.) de Janaúba-MG. In: Congresso Brasileiro de Processamento mínimo e Pós-colheita de frutas, flores e hortaliças, 001. Anais Congresso Brasileiro de Processamento mínimo e Pós-colheita de frutas, flores e hortaliças, Aracaju-SE.

Gomes, E. D.,Narain, N., Sa Ramalho, S. A., Gualberto, N. C., Miranda, R. M. \& Gomes, E. D. (2011). Development of a rapid isocratic reverse phase -ultra fast liquid chromatographic method for determination of phenolic acids in fruits. Planta Médica, 77, 12- 32. 10.1055/s-0031-1282208.

IAL (2008). Instituto Adolfo Lutz. Métodos físico-químicos para análise de alimentos. São Paulo: Intituto Adolfo Lutz, 1020 p. <http:www.ial.sp.gov.br/index.html>.

Lima, C. A., Faleiro, F. G., Junqueira, N. T. V., Cohen, K. O. \& Guimarâes, T. G. (2013). Características físico-químicas, polifenóis e flavonoides amarelos em frutos de espécies de pitaias comerciais e nativas do cerrado. Revista Brasileira de Fruticultura, 35(2). https://.org/10.1590/S0100-29452013000200027. 
Research, Society and Development, v. 10, n. 3, e42010313557, 2021

(CC BY 4.0) | ISSN 2525-3409 | DOI: http://dx.doi.org/10.33448/rsd-v10i3.13557

Moura, F. T., Silva, S. M., Schunemann, A. P. P. \& Martins, L. P. (2013). Frutos do umbuzeiro armazenados sob atmosfera modificada e ambiente em diferentes estádios de maturação. Revista Ciências Agronômicas, 44(4), 764 - 772. https://.org/10.1590/S1806-66902013000400013.

Moura, R. N., Ferreira, M. A. R., Passos, T. O., Lima, A. S., Castro, C. D. P. C., Rybka, A.C.P., Oliveira, V. R. \& Freitas, S. T. (2017). Qualidade físico-química e características biométricas de frutos de umbuzeiro do Banco de Germoplasma da Embrapa Semiárido. In: Jornada de Iniciação Científica da Embrapa Semiárido. Petrolina. Jornada de Iniciação Científica da Embrapa Semiárido.

Nascimento-Silva, O., Chinalia, L.A. \& Paiva, J. G. A. (2008). Caracterização histoquímica dos folíolos de Spondias tuberosa Arruda (Anacardiaceae L.). Revista Caatinga, Mossoró, 21(3), 62 - 68.

Oliveira, S. B., Azoubel, P. M. \& Araújo, A. J. (2008). Efeito de hidrocolóides na firmeza, atividade de água e sólidos solúveis de estruturado de polpa de umbu (Spondias tuberosa Arr. Cam.). In: Anais da $3^{a}$ Jornada de Iniciação Científica da Embrapa Semiárido, Petrolina, Brasil: Embrapa Semiárido, p. 201 - 208.

Passos, F. G., Cruz, R. G., Santos, M. V. \& Fernandes, R. V. B. (2013). Avaliação físico-química e sensorial de licores mistos de cenoura com laranja e maracujá. Revista Brasileira de Produtos Agroindustriais, Campina Grande, 15(3), 211-218.

Pereira, K. S., Leite, D. S., Santos, P. L. S. \& Cardoso, R. L. (2012). Preparo, caracterização físico-química e aceitabilidade de licor de corte de Spondias tuberosa. Enciclopédia Biosfera, 8(15), 1340.

Pinheiro, J. M. S., Rodrigues, M. L. M., Fonseca, S. N. A., Paraizo, E., A., Mizobutsi, G. P. \& Lopes, E. P. (2015). Caracterização física e química de frutos de umbu In: Congresso Brasileiro de Processamento mínimo e Pós-colheita de frutas, flores e hortaliças, 001. Anais. Aracaju-SE.

Ramalho, S. A., Gualberto, N. C., Oliveira, G. B., Gomes, E. D., Miranda, R. M. \& Narain, N. (2011). Evaluation of antioxidant capacity and L-ascorbic acid content in Brazilian tropical fruits acerola (Malpighia emarginata), mangaba (Harconia speciosa), siriguela (Spondias purpurea) and umbu (Spondias tuberosa). Planta Medica, 77, 200 - 212. https://.org/10.1055/s-0031-1282958.

Rufino, M. S. M., Alves, R. E., Brito, E. S., Pérez-Jiménez, J., Saura-Calixto, F. \& Mancini Filho, J. (2010). Bioactive compounds and antioxidant capacities of 18 non-traditional tropical fruits from Brazil. Food Chemistry, 121, 996 - 1002. https://.org/10.1016/j.foodchem.2010.01.037.

Silva, A. R. A., Morais, S. M., Marques, M. M. M., Lima, D. M., Santos, S. C. C., Almeida, R. R. \& Guedes, M. I. F. (2011). Antiviral activities of extracts and phenolic components of two Spondias species against dengue virus. The Journal of Venomous Animals and Toxins including Tropical Diseases, 17, 406 - 413, 2011. https://.org/10.1590/S1678-91992011000400007.

Silva, R. C., Amorim, A. B. F., Feitosa, R. M., Oliveira, E. N. A., Feitosa, B. F. \& Amadeu, L. T. S. (2017). Licor fino de casca de tangerina: processamento e caracterização. Arquivos brasileiros de alimentação, 2(3). http://.org/0000-0003-3172-4776.u.

Viera, V. B., Rodrigues, J. B., Brasil, C. C. B. \& Rosa, C. S. (2010). Produção, caracterização e aceitabilidade de licor de camu-camu (myrciaria dúbia (h.B.K.) Mcvaugh). Alimentos e. Nutrição, 21(4), 520.

Wang, S.Y. \& Zheng, W. (2003). Oxygen radical absorbing capacity of phenolics in blueberries, cranberries, chokeberries, and lingonberries. Journal of Agricultural and Food Chemistry. 51(2), 873 - 878. https://.org/10.1021/jf020728u. 